\title{
Comparison of Mental Health Service Utilization by Asian Americans and Non- Hispanic Whites versus Their Cardiovascular Care Utilization
}

\author{
Benjamin K. Woo ${ }^{1}$ \\ 1. UCLA, Los Angeles, Ca, UCLA, Los Angeles, CA
}

$\square$ Corresponding author: Benjamin K. Woo, bewoo@dhs.lacounty.gov

Disclosures can be found in Additional Information at the end of the article

\section{Abstract}

Introduction: This study will determine whether racial/ethnic disparities persist in various psychiatric disorders among Asian Americans.

Methods: Secondary analyses of data from the second largest public health system in the US (total $\mathrm{N}=22294$ ) were performed. Chi-squared statistics were used to compare the race for mental health service utilization for five psychiatric diagnoses. Cardiovascular care utilization by Asian Americans and non-Hispanic whites was used as a proxy for overall healthcare utilization rates between the two racial groups and constituted the expected values for the analysis.

Results: Asian Americans were less likely to utilize mental health services for bipolar disorder, schizophrenia, anxiety, depression, and intellectual disabilities.

Conclusion: The results of this study call for ways to increase mental health service utilization on par with cardiovascular healthcare utilization among Asian Americans.

Received 08/05/2017

Review began 08/17/2017

Review ended 08/18/2017

Published 08/22/2017

C) Copyright 2017

Woo. This is an open access article distributed under the terms of the

Creative Commons Attribution License CC-BY 3.0., which permits unrestricted use, distribution, and reproduction in any medium, provided the original author and source are credited.
Categories: Psychiatry, Public Health, Epidemiology/Public Health

Keywords: asians, asian americans, chinese americans, ethnic disparities, mental health service, health service utilization, schizophrenia

\section{Introduction}

Asian Americans are included as one of the major racial categories in the 2010 US Census [1]. The most recent census estimates show that there are 1.3 million Asian Americans in Los Angeles County, which accounts for $13.8 \%$ of the total county population. As this population continues to grow, healthcare providers must be cognizant of the considerable disparity in the recorded epidemiological prevalence of mental health issues and in mental health service utilization between Asian Americans and the general population [2-5].

The National Latino and Asian American Study (NLAAS) was the first national epidemiological survey of Asian Americans in the United States [6]. The study surveyed a national sample of 2095 Asian American individuals. The overall lifetime rate of Asian Americans having substance use disorders, anxiety disorders, or depression was 17.3\%. Data from the National Epidemiological Survey on Alcohol and Related Conditions (NESARC) revealed that the 12- 
month prevalence of any psychiatric disorder was $23.6 \%$ and $22.3 \%$ for Asian American males and females, respectively [5].

Despite the substantial risk for psychiatric symptoms among Asian Americans, racial disparities have been found in mental health service utilization by this group [2,7]. Data from the NLAAS revealed Asian Americans have lower rates of mental-health-related service use compared to the general population [2]. Additionally, a lower percentage of Asian Americans (34.1\%) with probable mental disorders sought any form of service as compared to their counterparts (41.1\%). The NESARC study showed that only 25\% of Asians with any Diagnostic and Statistical Manual of Mental Disorders - Fourth Edition (DSM-IV) psychiatric disorders utilized mental health services [7].

In the United States, the overall prevalence of hypertension among adults was $29 \%$ for the period 2011-2014 [8]. The prevalence of hypertension was lower among Asian Americans (24.9\%) than among non-Hispanic whites (28.0\%). In addition, the estimated prevalence of ischemic heart diseases (IHD) was lower among Asian Americans (4.3\%) as compared to 6.4\% of the total population [9]. Therefore, cardiovascular care utilization by Asian Americans and nonHispanic whites may serve as a proxy denominator to estimate the race/ethnicity distribution of a service area.

It is unclear whether ethnic/racial disparities persist in various psychiatric disorders among Asian Americans. Our primary goal was to measure overall ethnic/racial disparities by comparing mental health service utilization by Asian Americans and non-Hispanic whites versus their utilization of health-related service use for primary hypertension $(\mathrm{PH})$ and IHD. Our secondary goal was to measure ethnic/racial disparities by comparing the mental health service utilization of five psychiatric disorders by Asian Americans and non-Hispanic whites versus their utilization of PH health-related service use.

\section{Materials And Methods}

The study was carried out at the Los Angeles County's Department of Health Services (LACDHS). LACDHS is the second-largest public health system in the US and serves nearly 10 million residents. LACDHS includes four traditional hospital-based facilities as well as several off-site clinics. For the purpose of analysis, data derived from the LACDHS Electronic Health Record consists of a combined pool of patient visits to inpatient, emergency, and hospital outpatient facilities. The basic sampling unit is the physician-patient encounter or visit. Study participants included individuals of Asian or non-Hispanic white race/ethnicity between 18 and 65 years of age.

Data collected over a 30-month period (January 1, 2015 - June 30, 2017) included patient demographics, diagnoses, and various chronic conditions. We examined a subset of participants who had international classification of disease, tenth revision (ICD-10) codes for one of five psychiatric diagnoses: 1) Anxiety, dissociative, stress-related, somatoform, and other nonpsychotic mental disorders (F40-F48); 2) Bipolar disorders or manic episodes (F30-F31); 3) Intellectual disabilities (F70-F79); 4) Major depressive disorders (F32-F33); and 5) Schizophrenia, schizotypal, delusional, and other non-mood psychotic disorders (F20-F29). A comparison group with primary hypertension (I10) was selected to determine disparities in mental health service utilization. The race-proportions of visits for $\mathrm{PH}$ were used as the expected values in the chi-squared analyses. A separate analysis was then performed using the race-proportions of visits for ischemic heart diseases (I20-I25) as a comparison group to confirm the overall ethnic/racial disparities.

Data collected from the LACDHS electronic health record were analyzed with chi-squared statistics to compare the effect of race/ethnicity on mental health service utilization for each of 


\section{Cureus}

the five psychiatric diagnoses with the effect of race/ethnicity on service utilization for $\mathrm{PH}$. This research was deemed exempt research involving human subjects by the LACDHS, as it utilized pre-existing, de-identified data. Data were entered and analyzed using IBM SPSS Statistics v. 21.0 (Armonk, NY).

\section{Results}

Of the 22294 psychiatric health service visits in the study, 2735 (12.3\%) visits were from Asian Americans and 19559 (87.7\%) visits were from non-Hispanic Whites. There were 11129 (49.9\%) females and 11165 (50.1\%) males. The study included 8444 (37.9\%) cases aged 18-34 years old, 4399 (19.7\%) cases aged 35-44 years old, 4673 (21.0\%) cases aged $45-64$ years old, and 4778 (21.4\%) cases aged 55-64 years old. Table 1 presents descriptive statistics for health service visits relating to each of the five psychiatric diagnoses.

\begin{tabular}{|c|c|c|c|c|c|c|c|c|c|c|c|c|}
\hline & \multicolumn{2}{|c|}{$\begin{array}{l}\text { Schizophrenia (F20- } \\
\text { F29) }\end{array}$} & \multicolumn{2}{|c|}{$\begin{array}{l}\text { Bipolar Disorders } \\
\text { (F30-F31) }\end{array}$} & \multicolumn{2}{|c|}{$\begin{array}{l}\text { Depression (F32- } \\
\text { F33) }\end{array}$} & \multicolumn{2}{|c|}{$\begin{array}{l}\text { Anxiety (F40- } \\
\text { F48) }\end{array}$} & \multicolumn{2}{|c|}{$\begin{array}{l}\text { Intellectual Disabilities } \\
\text { (F70-F79) }\end{array}$} & \multirow{2}{*}{$\begin{array}{l}\text { Total } \\
(n=2229\end{array}$} & \\
\hline & $(n=5437)$ & & $(n=2011)$ & & $(n=6629)$ & & $(n=7742)$ & & $(n=475)$ & & & \\
\hline \multicolumn{13}{|l|}{ Gender } \\
\hline Female & 1853 & $34.1 \%$ & 977 & $48.6 \%$ & 3726 & $56.2 \%$ & 4356 & $56.3 \%$ & 215 & $45.3 \%$ & 11129 & $49.9 \%$ \\
\hline Male & 3584 & $65.9 \%$ & 1034 & $51.4 \%$ & 2903 & $43.8 \%$ & 3386 & $43.7 \%$ & 260 & $54.7 \%$ & 11169 & $50.1 \%$ \\
\hline \multicolumn{13}{|c|}{ Living status } \\
\hline Living & 5425 & $99.8 \%$ & 2005 & $99.7 \%$ & 6605 & $99.6 \%$ & 7709 & $99.6 \%$ & 471 & $99.2 \%$ & 22215 & $99.6 \%$ \\
\hline Deceased & 12 & $0.2 \%$ & 6 & $0.3 \%$ & 24 & $0.4 \%$ & 33 & $0.4 \%$ & 4 & $0.8 \%$ & 79 & $0.4 \%$ \\
\hline \multicolumn{13}{|c|}{ Race/Ethnicity } \\
\hline Asian & 572 & $10.5 \%$ & 185 & $9.2 \%$ & 913 & $13.8 \%$ & 995 & $12.9 \%$ & 70 & $14.7 \%$ & 2735 & $12.3 \%$ \\
\hline Whites & 4865 & $89.5 \%$ & 1826 & $90.8 \%$ & 5716 & $86.2 \%$ & 6747 & $87.1 \%$ & 405 & $85.3 \%$ & 19559 & $87.7 \%$ \\
\hline \multicolumn{13}{|l|}{ Age group } \\
\hline 18-34 & 2372 & $43.6 \%$ & 768 & $38.2 \%$ & 2067 & $31.2 \%$ & 2952 & $38.1 \%$ & 285 & $60.0 \%$ & 8444 & $37.9 \%$ \\
\hline $35-44$ & 1225 & $22.5 \%$ & 447 & $22.2 \%$ & 1091 & $16.5 \%$ & 1555 & $20.1 \%$ & 81 & $17.1 \%$ & 4399 & $19.7 \%$ \\
\hline $45-64$ & 1051 & $19.3 \%$ & 442 & $22.0 \%$ & 1544 & $23.3 \%$ & 1567 & $20.2 \%$ & 69 & $14.5 \%$ & 4673 & $21.0 \%$ \\
\hline $55-64$ & 789 & $14.5 \%$ & 354 & $17.6 \%$ & 1927 & $29.1 \%$ & 1668 & $21.5 \%$ & 40 & $8.4 \%$ & 4778 & $21.4 \%$ \\
\hline
\end{tabular}

\section{TABLE 1: Demographics of cases with the five psychiatric diagnoses}

1) Source of Data: Los Angeles County's Department of Health Services (LACDHS) electronic health record.

2) International classification of disease, tenth revision (ICD-10) codes were provided underneath each one of the five psychiatric diagnoses. 
For PH health-related service use, there were 5473 (25.0\%) Asian American cases and 16428 (75.0\%) non-Hispanic white cases. Compared to non-Hispanic whites, Asian Americans were significantly less likely to utilize any mental health services, even when accounting for changes in health care utilization (using PH health-related service use as a proxy) that already exist between races (chi-square $=1182.34, \mathrm{df}=1, \mathrm{p}<.01$ ). This significant trend exists across the continuum of mental health disorders: Asian Americans were less likely to utilize mental health services for anxiety disorders (chi-square $=494.00, \mathrm{df}=1, \mathrm{p}<.01$ ), bipolar disorders (chisquare $=254.25, \mathrm{df}=1, \mathrm{p}<.01$ ), intellectual disabilities (chi-square $=26.23, \mathrm{df}=1, \mathrm{p}<.01$ ), major depressive disorders (chi-square $=368.53, \mathrm{df}=1, \mathrm{p}<.01$ ), and schizophrenia (chi-square $=529.48$, $\mathrm{df}=1, \mathrm{p}<.01)$.

For IHD health-related service use, there were 575 (22.8\%) Asian American cases and 1945 (77.2\%) non-Hispanic white cases. Using IHD-related utilization as a proxy to estimate the race/ethnicity distribution of the service area, Asian Americans, in comparison to non-Hispanic whites, were significantly less likely to utilize any mental health services (chi-square=217.98, $\mathrm{df}=1, \mathrm{p}<.01)$.

\section{Discussion}

The present study explored the racial disparities in the delivery of care to patients with psychiatric disorders as compared to patients with PH. Significant differences were seen in overall mental health service use, with less Asian Americans than non-Hispanic whites receiving mental health service even when compared to their respective hypertension healthcare service utilization. This finding was confirmed with IHD health-related service use as another proxy to estimate the race/ethnicity distribution of the denominator population. This pattern is consistent with previous reports on ethnic disparities between Asian Americans and non-Hispanic whites [2,7]. Nonetheless, the implications and reasons for such ethnic disparities require further inquiry to ensure an equal quality of care for vulnerable populations.

Despite comparable rates of mental health disorders and overall disease among Asian Americans and non-Hispanic whites, this study revealed that while $25.0 \%$ of hypertension health care was utilized by Asian Americans, they only accounted for 9.2\%, 10.5\%, 12.9\%, 13.8\%, and $14.7 \%$ of mental health service utilization for bipolar disorder, schizophrenia, anxiety, depression, and intellectual disabilities, respectively. Particularly for Asian Americans, stigma and other barriers can deter patients and their families from seeking needed mental health services [10-11]. In comparison with medical illnesses, the stigma surrounding psychiatric disorder contributes to shame in the Asian American community, which can affect both quality and access to care [12-13]. Our results further highlight that among psychiatric disorders, Asian Americans with schizophrenia or bipolar disorders were less likely to utilize mental health service. Innovations in delivering culturally sensitive health education are needed to reduce such disparities in health and health care [14-15].

This study has several limitations. First, the nature of pre-existing, de-identified data of one county impacts its generalizability. Second, without individual-level data, the study did not account for other factors that have shown to determine racial disparities. Third, we have no detailed information on the mental health visits. Fourth, the study did not account for racial/ethnic differences in care-seeking for PH and IHD. The observation of different race/ethnicity distribution could actually have more to do with disparities in cardiac issues than with disparities in mental health care. Future research will need to be performed to further delineate these relationships. Finally, the dataset did not distinguish among Asian American subgroups; it is important to acknowledge that Asian Americans comprise a heterogeneous group. Despite these limitations, our study calls for ways to increase mental health service utilization on par with cardiovascular healthcare utilization among Asian Americans. 


\section{Conclusions}

Asian Americans were less likely to utilize mental health services for bipolar disorder, schizophrenia, anxiety, depression, and intellectual disabilities. The results of this study call for ways to increase mental health service utilization on par with cardiovascular healthcare utilization among Asian Americans.

\section{Additional Information \\ Disclosures}

Human subjects: Consent was obtained by all participants in this study. Animal subjects: All authors have confirmed that this study did not involve animal subjects or tissue. Conflicts of interest: In compliance with the ICMJE uniform disclosure form, all authors declare the following: Payment/services info: All authors have declared that no financial support was received from any organization for the submitted work. Financial relationships: All authors have declared that they have no financial relationships at present or within the previous three years with any organizations that might have an interest in the submitted work. Other relationships: All authors have declared that there are no other relationships or activities that could appear to have influenced the submitted work.

\section{References}

1. 2010 Census Shows Asians are Fastest-Growing Race Group. (2012). Accessed: August 15, 2017: https://www.census.gov/newsroom/releases/archives/2010_census/cb12-cn22.html.

2. Abe-Kim J, Takeuchi DT, Hong S, et al.: Use of mental health-related services among immigrant and US-born Asian Americans: results from the National Latino and Asian American Study. Am J Public Health. 2007, 97:91-98. 10.2105/AJPH.2006.098541

3. Takeuchi DT, Chung RC, Lin KM, Shen H, Kurasaki K, Chun CA, Sue S: Lifetime and twelvemonth prevalence rates of major depressive episodes and dysthymia among Chinese Americans in Los Angeles. Am J Psychiatry. 1998, 155:1407-1414. 10.1176/ajp.155.10.1407

4. Woo BK, Lo TT: How often do Chinese Americans stay on treatment after transitioning from outpatient mental health to primary care setting. Gen Hosp Psychiatry. 2011, 33:e5-e6. 10.1016/j.genhosppsych.2010.09.002

5. Xu Y, Okuda M, Hser YI, Hasin D, Liu SM, Grant BF, Blanco C: Twelve-month prevalence of psychiatric disorders and treatment-seeking among Asian Americans/Pacific Islanders in the United States: results from the National Epidemiological Survey on Alcohol and Related Conditions. J Psychiatr Res. 2011, 45:910-918. 10.1016/j.jpsychires.2010.12.009

6. Takeuchi DT, Zane N, Seunghye H, et al.: Immigration-related factors and mental disorders among Asian Americans. Am J Public Health. 2007, 97:84-90. 10.2105/AJPH.2006.088401

7. Lee SY, Martins SM, Lee HB: Mental disorders and mental health service use across Asian American subethnic groups in the United States. Community Ment Health J. 2015, 51:153160. 10.1007/s10597-014-9749-0

8. Hypertension Prevalence and Control Among Adults: United States, 2011-2014. (2015). Accessed: August 15, 2017: https://www.cdc.gov/nchs/products/databriefs/db220.htm.

9. Go AS, Mozaffarian D, Roger VL, et al.: Heart disease and stroke statistics - 2013 update: a report from the American Heart Association. Circulation. 2013, 127:e6-e245. 10.1161/CIR.0b013e31828124ad

10. Woo BK: Dementia health promotion for Chinese Americans . Cureus. 2017, 9:e1411. doi:10.7759/cureus.1411

11. Diamond AG, Woo BK: Duration of residence and dementia literacy among Chinese Americans. Int J Soc Psychiatry. 2014, 60:406-409. 10.1177/0020764013491742

12. Woo BK, Mehta P: Examining the differences in the stigma of dementia and diabetes among Chinese Americans. Geriatr Gerontol Int. 2017, 17:760-764. 10.1111/ggi.12782

13. Woo BK: Family history and its relationship with dementia stigma beliefs among Chinese Americans. Geriatr Gerontol Int. 2017, 17:122-125. 10.1111/ggi.12686

14. Zheng X, Woo BK: E-mental health in ethnic minority: A comparison of youtube and talk- 


\section{Cureus}

based educational workshops in dementia. Asian J Psychiatr. 2017, 25:246-248.

10.1016/j.ajp.2016.12.002

15. Zheng X, Chung JO, Woo BK: Exploring the impact of a culturally tailored short film in modifying dementia stigma among Chinese Americans: a pilot study. Acad Psychiatry. 2016, 40:372-374. 10.1007/s40596-015-0397-7 